\title{
Tandem Mass Spectrometric Analysis of a Mixture of Isobars Using the Survival Yield Technique
}

\author{
Antony Memboeuf, Laure Jullien, Rémy Lartia, Bernard Brasme, Yves Gimbert \\ Département de Chimie Moléculaire, UMR 5250-ICMG-FR 2607, CNRS Université Joseph Fourier, BP 53, 38041 Grenoble, \\ Cedex 9, France
}

\begin{abstract}
Collision induced dissociation tandem mass spectrometry experiments were performed to unequivocally separate compounds from an isobaric mixture of two products. The Survival Yield curve was obtained and is shown to consist in a linear combination of the curves corresponding to the two components separately. For such a mixture, a plateau appears on the diagram in lieu of the continuous decrease expected allowing for the structural study of the two components separately. The width of the plateau critically relates to the fragmentation parameters of the two molecular ions, which need to be sufficiently different structurally for the plateau to be observed. However, at constant fragmentation parameters, we have observed the width significantly increases at large $\mathrm{m} / \mathrm{z}$. This makes the separation more and more efficient as isobars have larger $\mathrm{m} / \mathrm{z}$ and the technique complementary to those applicable at low $\mathrm{m} / \mathrm{z}$ only. We have observed that the vertical position of the plateau relates linearly to the relative concentration of the two compounds that may be useful for quantification. Repeatability was estimated at $2 \%$ on a quadrupole ion trap. An advantage of using survival yield curves only, is that a priori knowledge of the respective fragmentation patterns of the two isobars becomes unnecessary. Consequently, similar performances are obtained if fragments are isobaric, which is also demonstrated in our study. The critical case of reverse peptides, at low $\mathrm{m} / \mathrm{z}$ and similar fragmentation parameters, is also presented as a limitation of the method.
\end{abstract}

Key words: Isobar, Fragmentation, Quantification, Structural analysis, Survival yield technique, Tandem mass spectrometry, Polyether, Contamination

\section{Introduction}

$\mathrm{T}$ here is a growing demand on mass spectrometric techniques for high-throughput analysis, to perform more reliably and provide more information on increasingly complex samples: the analysis of compounds in biological samples [1], in the field of petroleomics [2, 3], to study isomers $[4,5]$, or simply for separating unresolved peaks due to technical limitations [6]. With this respect, the identification and quantification of isobars remain challenging tasks

Electronic supplementary material The online version of this article (doi:10.1007/s13361-011-0195-8) contains supplementary material, which is available to authorized users.

Correspondence to: Antony Memboeuf; e-mail: a.memboeuf@laposte.net for mass spectroscopists. The identification and, possibly, elimination of an isobar becomes essential in order to perform the structural analysis, ultimately to quantify, a compound of interest. This may require tedious preparative work, coupling with chromatographic separation techniques or more recently ion mobility spectrometry, possibly combined with complex procedures [7,8]. A simpler and faster alternative consists of using collision induced dissociation tandem mass spectrometry experiments combined with the kinetic method. Using this technique, mixtures of chiral or isomeric peptides may bind to transition metals, allowing for quantitative analysis [9, 10]. This is, however, limited to low mass compounds. A number of techniques utilizing branching ratios of product ions were also developed $[6,11,12]$. Alternatively, MS/MS spectra obtained at 
"low" and at "high" collision energies may be used to make this structural analysis. The latter procedures rely on the fact that fragment ions of the two isobars are formed at different collision energies according to differences in the structures of the isobaric components. More precisely, different fragmentation parameters (activation energy and entropy) may be associated with the formation of fragment ions due to differences in the nature of the fragmentation or dissociation processes. However, when monitoring branching ratios, one needs to have an a priori knowledge of MS/MS spectra for both isobars. Moreover, in the case fragment ions are also isobaric, tandem MS technique does not allow even for the qualitative identification of the presence of two components. In this paper, we present a simple MS/MS based method, using the survival yield technique, to overcome these issues. This allows for qualitative identification of the mixture, even when fragments are also isobars, without a priori knowledge of fragment ions. MS/MS spectra for two isobaric homopolymers obtained at relatively large polymerization degree are presented both separately and in mixtures. The main features observed are discussed in detail, in particular which structural information can be obtained for both components from this procedure. The possibility for quantitative analysis is presented, as well as the performance of the method and how it is affected by mass to charge ratio. Finally, a critical case consisting of two reverse peptides is also discussed in detail.

\section{Experimental}

\section{Chemicals}

Samples of synthetic polymers poly(lactic acid) -PLA, HO$\left(\mathrm{C}_{3} \mathrm{H}_{4} \mathrm{O}_{2}\right)_{\mathrm{n}}-\mathrm{H}-$, and poly(tetramethylene glycols) -PTMEG, Terathane or PTHF for poly(tetrahydrofuran), HO- $\left(\mathrm{C}_{4} \mathrm{H}_{8} \mathrm{O}\right)_{\mathrm{n}^{-}}$ $\mathrm{H}-$ both with number-average molecular weight $1000 \mathrm{~g} / \mathrm{mol}$ were used. PTMEG was purchased from Sigma-Aldrich (Schnelldorf, Germany) and PLA was kindly provided by J. De Winter (Mass Spectrometry Research Group of UMONS, Mons, Belgium). Peptides with inverted sequences $\mathrm{H}-$ (SDGRGAF)- $\mathrm{NH}_{2}$ (reverse 1) and H-(FAGRGDS)- $\mathrm{NH}_{2}$ (reverse 2) were synthesized in our lab (Nanobio Platform). They were assembled on an ABI433 peptides synthesizer following Fmoc strategy. The following side-chain protected amino acids were used: $\operatorname{Ser}(\mathrm{tBu}) ; \operatorname{Arg}(\mathrm{pbf})$; and $\mathrm{Asp}(\mathrm{tBu})$. Rink amide resin, loaded to $0.79 \mathrm{mmol} / \mathrm{g}$, was used. Synthesis were performed on a $0.1 \mathrm{mmol}$ scale using 10 equivalents amino acids dissolved in $2 \mathrm{~mL}$ NMP, 9.5 equivalents HBTU and 20 equivalents DIEA. Deprotection was performed by a NMP/ piperidine (4:1) solution and monitored by UV. Final deprotection was achieved by shaking resin in $50 \mathrm{~mL}$ TFA/ $\mathrm{H}_{2} \mathrm{O} / \mathrm{TIS}$ (95:2.5:2.5) for $2 \mathrm{~h}$. Deprotection solution was evaporated to dryness and the resulting oily residue was precipitated in $\mathrm{Et}_{2} \mathrm{O}$ $(40 \mathrm{~mL})$ and centrifuged. White precipitate was washed by $\mathrm{Et}_{2} \mathrm{O}$ $(3 \times 30 \mathrm{~mL})$. Crude mixture was purified by RP-HPLC on a Gilson GX-281 apparatus. Eluent A is a 0.1\% TFA solution and eluent $\mathrm{B}$ is $0.1 \%$ TFA solution in $\mathrm{MeCN} / \mathrm{H}_{2} \mathrm{O}(9: 1)$. Peptide reverse 1 was separated from small amount of $\beta$-branched peptide resulting from aspartimide formation using the following gradient: $10 \%$ of eluent B for $2 \mathrm{~min}$, followed by $10 \%$ to $40 \%$ in $15 \mathrm{~min}$. Reverse 1 peptide was isolated at $10.94 \mathrm{~min}$ and base-to-base resolved from its main impurity $\left(t_{R}=11.30 \mathrm{~min}\right)$. Peptide reverse 2 was purified using the following gradient: $0 \%$ of eluent B for $2 \mathrm{~min}$, followed by $0 \%$ to $100 \%$ in $15 \mathrm{~min}$. Peptide was isolated at $8.60 \mathrm{~min}$. Fractions were gathered and lyophilized to afford white powder in ca. $60 \%$ yield.

\section{ESI Quadrupole Ion Trap MS/MS Experiments}

The experiments were performed on a Bruker Esquire 3000plus ion trap mass spectrometer (Bruker Daltonics, Bremen, Germany). Samples of synthetic polymers were dissolved in pure $\mathrm{MeOH}$ at $5.10^{-5} \mathrm{M}, \mathrm{LiCl}$ was added at $10^{-}$ ${ }^{5} \mathrm{M}$. Peptides were dissolved in water/acetonitrile 1:1 with $0.1 \%$ formic acid to generate protonated signal. The settings of the instrument were the following: capillary voltage $2 \mathrm{kV}$; nitrogen nebulizer pressure $11 \mathrm{psi}$; nitrogen dry gas flow $5 \mathrm{~L} / \mathrm{min}$; dry gas temperature $300^{\circ} \mathrm{C}$; He collision gas was used at an average pressure in the ion trap of $1.25 \times 10^{-}$ ${ }^{5}$ mbar (uncorrected gauge reading). The number of trapped ions was approximately 10,000 and was kept constant by using the ion charge control (ICC) option throughout all measurements. Isolation was performed using a $0.8 \mathrm{Th}$ window and followed immediately by a $50 \mathrm{~ms}$ cooling period (fragmentation delay). Molecular ions were fragmented by applying an excitation voltage during $100 \mathrm{~ms}$ (fragmentation time) over a $10 \mathrm{Th}$ mass to charge window (fragmentation width). SmartFrag option was turned off and the $27 \%$ default mass cut-off was applied. Recorded mass spectra were analyzed using Data Analysis 3.3 software (Bruker Daltonics, Bremen, Germany). During tandem MS (MS/MS) experiments, 1 min acquisition was performed at each excitation voltage. For an improved statistic and repeatability, data are further summed up over the last $30 \mathrm{~s}$ of the acquisition stage in order to generate a MS/MS spectrum from which peaks intensities can be further extracted. For accurate quantitative analysis the duration of acquisition may be increased and data summed up over $1 \mathrm{~min}$ or more in order to reduce error bars.

\section{Results and Discussions}

\section{MS/MS Spectra of Lithium Cationized PLA and PTMEG and of Their Mixture}

We have performed tandem mass spectrometric analysis of lithium cationized PLA and PTMEG homopolymers, both separately and in mixtures. These homopolymers have different structures but monomers are essentially isobaric. Our analysis was performed with degree of polymerization varying from 14 up to 23 . The corresponding $\mathrm{m} / \mathrm{z}$ range goes approximately from $\mathrm{m} / \mathrm{z} 1000$ up to $\mathrm{m} / \mathrm{z} 1700$, and has been selected to avoid overlapping of multiply charged ion 
distributions, which would complicate the picture, and to assess the technique on large mass to charge ratio. In Figure 1, tandem mass spectra were obtained at two excitation voltages: $0.8 \mathrm{~V}$ (spectra $\mathrm{d}$, e, and $\mathrm{f}$ ) and $1.3 \mathrm{~V}$ (spectra a, b, and c). They were obtained for pure homopolymers, $\left[\mathrm{PLA}_{23}+\mathrm{Li}\right]^{+}$(spectra a and $\mathrm{d}$ ) and $\left[\mathrm{PTMEG}_{23}+\mathrm{Li}\right]^{+}$(spectra $\mathrm{c}$ and $\mathrm{f}$ ), and for a 5:1 mixture of PLA/PTMEG (spectra b and e). A 5:1 ratio was found more suitable for illustrative purposes; a detailed analysis of this aspect is presented in the following section discussing quantitative information. For convenience, and without loss of information, these spectra are presented on the same mass range going from $\mathrm{m} / \mathrm{z} 1350$ up to $\mathrm{m} / \mathrm{z} 1700$; spectrum for $\left[\mathrm{PLA}_{23}+\mathrm{Li}\right]^{+}$is flat outside this $\mathrm{m} / z$ range and the same series of peaks for $\left[\mathrm{PTMEG}_{23}+\mathrm{Li}\right]^{+}$extend down to approximately $\mathrm{m} / \mathrm{z} 1000$ from which fragment ions are minor. Spectra a and c clearly exhibit similarities; indeed, the same series of peaks regularly spaced by $72 \mathrm{Th}$ from molecular ions peak is observed, although peak intensities from $\left[\mathrm{PLA}_{23}+\mathrm{Li}\right]^{+}$tend to decrease at low $\mathrm{m} / z$. One also notices an additional series present in the MS/MS spectrum of $\left[\mathrm{PTMEG}_{23}+\mathrm{Li}\right]^{+}$that is actually missing for $\left[\mathrm{PLA}_{23}+\mathrm{Li}\right]^{+}$ and is shifted from the aforementioned series by $-18 \mathrm{Th}$ (for detailed discussions of fragmentation mechanisms and MS/MS spectra interpretations, one may refer to recent publications $[13,14])$. However, in the case of a mixture of PTMEG samples with PLA, which we may refer to as contamination throughout the discussion, visual inspection is not sufficient to distinguish between fragment ions (spectrum $b$ in Figure 1). Clearly, in spite of their structural differences, tandem mass spectra of this mixture of homopolymers do not allow even for a qualitative identification of contamination. At this point, it is important to note the large difference in the degree of fragmentation achieved between the two homopolymers at the same excitation voltage: $0.8 \mathrm{~V}$ almost the entire population of $\left[\mathrm{PLA}_{23}+\mathrm{Li}\right]^{+}$molecular ions have already fragmented (spectrum $\mathrm{d}$ ), when, on the contrary, tandem mass spectrum $\mathrm{f}$ of $\left[\mathrm{PTMEG}_{23}+\mathrm{Li}\right]^{+}$does not exhibit a single fragment ion. This striking difference suggests the fragmentation parameters (activation energy and entropy) are significantly different and that these homopolymers may be separated according to this criterion.

\section{Structural Analysis of Isobaric Compounds in a Mixture Using the Survival Yield Technique}

The survival yield technique uses survival yield (SY) ratio, which is a convenient quantitative measure of the fragmentation degree that is defined according to:

$$
S Y=\frac{I_{M}}{I_{M}+\sum I_{F}}
$$

where $I_{F}$ stands for the intensities of fragment ions peaks and $I_{M}$ for the molecular ions peak intensity. Using this definition, one may record and plot the value of this ratio as a function of the excitation voltage: this leads to the SY curve. This technique has already been used in fundamental studies [15-18], but also to discriminate molecular ions according to their structures [19-21]. When doing so in the same experimental conditions for both homopolymers separately and for a mixture of 5:1 (PLA/PTMEG), one obtains the curves displayed in Figure 2. In the case of a pure homopolymer, the SY curve has the shape of a sigmoid starting from 1 at low excitation voltages (no fragment ions observed) decreasing continuously to 0 at higher excitation voltages (no molecular ions remain). An important point is that at the same molecular ion mass, the SY curve obtained for lithium cationized PTMEG is shifted to much higher excitation voltages compared with the one obtained for PLA. This is in agreement with our recent observation that polyethers do fragment at significantly higher excitation voltages compared with polyesters [19]. On the contrary, the SY curve obtained for a mixture of the two samples exhibits a plateau in place of the continuous decrease with the excitation voltage. After the $\left[\mathrm{PLA}_{23}+\mathrm{Li}\right]^{+}$component has fully fragmented to form fragment ions (at approximately $0.8 \mathrm{~V}$ according to the curve obtained for pure $\left[\mathrm{PLA}_{23}+\mathrm{Li}\right]^{+}$), only lithium cationized PTMEG molecular ions remain and do not fragment until a $1 \mathrm{~V}$ excitation voltage is applied. Consequently, from 0.8 up to $1 \mathrm{~V}$, the excitation process does not lead to the formation of additional fragments, and the ratio expressed in Eq. (1) remains constant on that range of excitation voltages. Starting from $1 \mathrm{~V}$, the SY ratio starts to decrease again as $\left[\mathrm{PTMEG}_{23}+\mathrm{Li}\right]^{+}$is fragmenting, to finally reach 0 value at high excitation voltages (complete fragmentation of both cationized homopolymers).

Following this reasoning, three regimes can be identified: a "low excitation regime" with spectra obtained at excitation voltages lower than $0.8 \mathrm{~V}$, an "intermediate excitation regime" that consists in a plateau (from 0.8 up to $1 \mathrm{~V}$ ) and, a "high excitation regime" corresponding to the second decrease of the SY curve from 1 up to $1.4 \mathrm{~V}$. Combining this nomenclature with previous qualitative arguments, the content of structural information of tandem MS spectra obtained at each regime may now be discussed in more detail. Fragment ions that are formed at the low and at the intermediate excitation regimes are obviously produced by the first component only, that is $\left[\mathrm{PLA}_{23}+\mathrm{Li}\right]^{+}$. Hence, MS/ MS spectra are characteristic of this isobar only and, by doing mass-selection of fragment ions obtained at voltages lower than $1 \mathrm{~V}$, multistage mass spectrometry can be performed on this component only, avoiding isobaric contamination and ambiguous structural analysis. Complementarily, molecular ions surviving the excitation process at the intermediate and at the high excitation regimes are clearly of the second type, that is $\left[\mathrm{PTMEG}_{23}+\mathrm{Li}\right]^{+}$. This is obviously the case as the entire population of molecular ions of the first component has been discarded from the MS/MS spectrum through fragmentation. Similarly, a second isolation stage of molecular ions may then be performed to study the structure of the second component as if there were no isobaric 
$=$ (a)

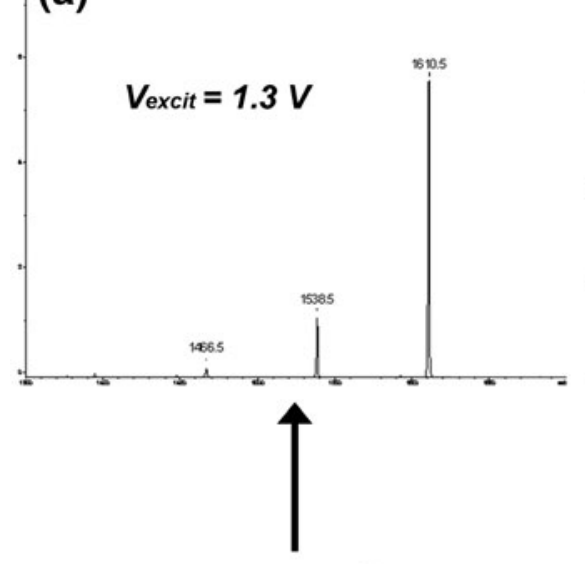

$\left[\mathrm{Li}_{-\mathrm{PLA}}\right]^{+}$

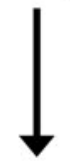

$\Rightarrow$ (d)

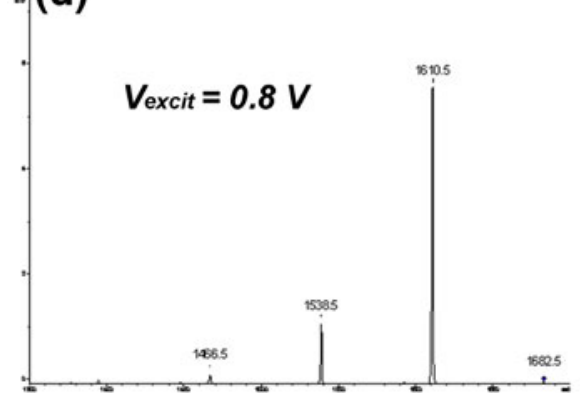

(b)

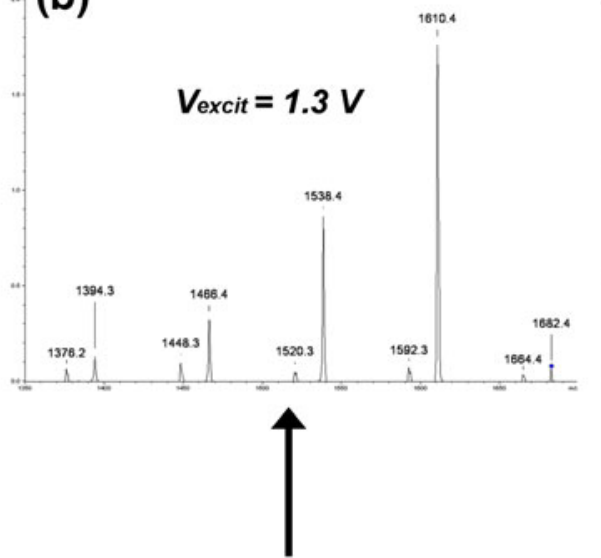

Mixture

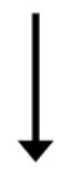

(e)

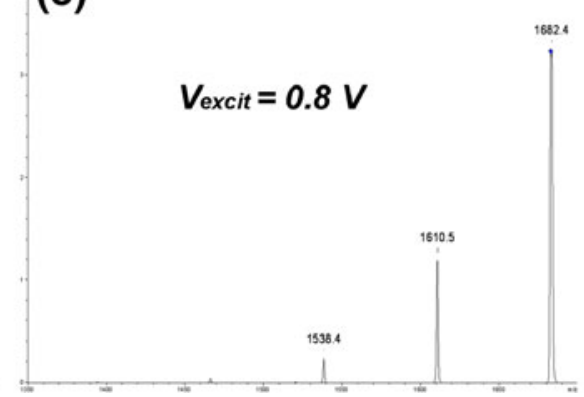

- (c)

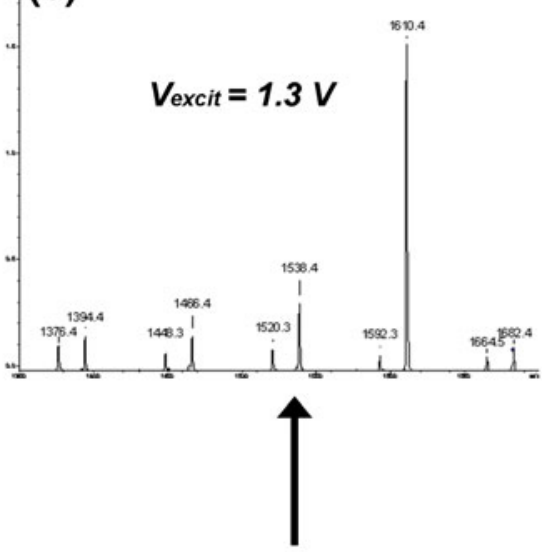

[Li-PTMEG $\left.{ }_{23}\right]^{+}$

(f)

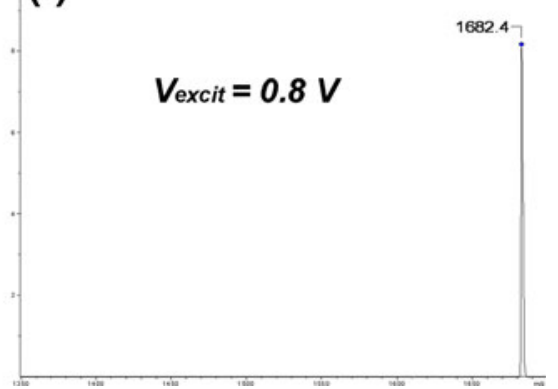

Figure 1. MS/MS spectra for $\left[\mathrm{PLA}_{23}+\mathrm{Li}^{+}\right.$(spectra $\mathbf{a}$ and $\mathbf{d}$ ), $\left[\mathrm{PTMEG}_{23}+\mathrm{Li}^{+}\right.$(spectra $\mathbf{c}$ and $\mathbf{f}$ ) and a $5: 1$ mixture (spectra $\mathbf{b}$ and $\mathbf{e}$ ), obtained at $0.8 \mathrm{~V}$ (spectra $\mathbf{d}, \mathbf{e}$, and $\mathbf{f}$ ) and $1.3 \mathrm{~V}$ (spectra $\mathbf{a}, \mathbf{b}$, and $\mathbf{c}$ ) excitation voltages

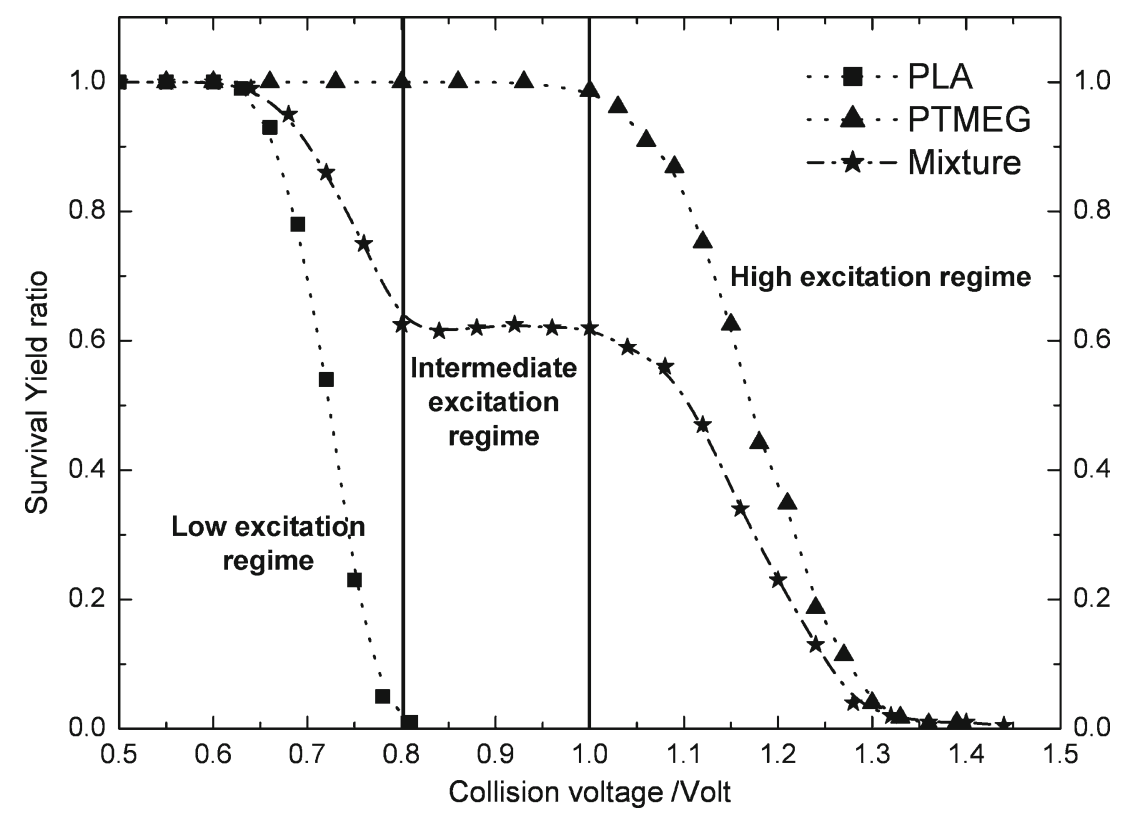

Figure 2. Survival Yield curves for $\left[\mathrm{PLA}_{23}+\mathrm{Li}^{+}\right.$(filled squares with dotted line) and $\left[\mathrm{PTMEG}_{23}+\mathrm{Li}^{+}\right.$(filled triangles with dotted line) and a 5:1 mixture of $\left[\mathrm{PLA}_{23}+\mathrm{Li}^{+} /\left[\mathrm{PTMEG}_{23}+\mathrm{Li}^{+}\right.\right.$(filled stars with dot dashed line) 
contaminant. Clearly, using this technique, separation and structural analysis of isobars can be performed using multistage mass spectrometric experiments only. Results are clearly unequivocal and do not depend on the a priori knowledge of the SY curves of pure analytes. However, the presented strategies critically rely on the identification of a plateau that enables clearly distinguishing between excitation regimes.

\section{Quantification of Contaminant and Tandem Mass Spectra Correction}

An important feature is that at the intermediate regime, one simultaneously observes fragment ions of the first component and molecular ions of the second. Therefore, after an excitation voltage corresponding to this regime has been applied, between 0.8 and $1 \mathrm{~V}$ in our case, the two isobars are completely separated. By reckoning the ratio of molecular ions peak intensity over the sum of fragment ions peaks intensities (more rigorously, the value of the SY ratio normalized to its maximum value, which is simply unity), one would thus expect the relative proportion of the two components to be unambiguously determined. To assess this anticipation, mixtures of PLA/PTMEG with ratio 1:1, 3:1, 5:1, 7:1, 8:1, 9:1, and 11:1 were prepared, and the corresponding SY curves measured to be compared with SY curves for pure oligomers (ratio 1:0 and 0:1). Results are presented in Figure 3. Clearly, increasing the quantity of PLA relative to PTMEG leads to a shift of the plateau to lower values of the SY ratio, which feature does agree with our previous understanding of the SY curve behavior. Furthermore, this qualitative finding can be supplemented by quantitative estimations: the inverse of SY ratio can be linearly related with the ratio of the two components (inset of Figure 3) with very good approximation. The correlation coefficient $\mathrm{R}^{2}$ was found to be 0.983 if the curve was enforced to go through $(0 ; 1)$ and $\mathrm{R}^{2}=0.992$ with a standard deviation of 0.041 when the intercept was allowed to vary (in such case the curve goes through $y=0.94$ in place of 1 ). The value of the intercept corresponds to the position of the SY curve in the absence of the first contaminant (that is for a sample of pure PTMEG), it is thus a good sign as it goes smoothly through this value. The same linearity would be obtained by plotting the SY ratio as a function of PTMEG/PLA ratio (in place of PLA/PTMEG), since the position of the plateau (brutto value) corresponds to the amount of the second component (PTMEG) relative to the amount of the first component (PLA). One may also note there is not a one to one correspondence between the SY ratio at the plateau and the PLA/PTMEG quantities introduced in the mass spectrometer. For example, when a 1:1 mixture of PLA/PTMEG was analyzed, the SY ratio determined was close to 0.92 . The slope of the linear fit with intercept $(0 ; 1)$ is 0.164 , when one would appreciate to have unity for straight quantitative analysis. Obviously differences in the lithium cation affinity, in the ionization or isolation efficiency or in the excitation efficiency (theoretically, there is $0.2 \mathrm{Th}$ difference between these two isobars) may be responsible for this discrepancy. Moreover, controlling the initial concentration for single oligomers is not feasible as the concentration reflects only the number-average molecular weight of the polymer mass distribution not the one of a single oligomer. In spite of all these complications, we still observe a linear tendency that is very encouraging, and allows for using this methodology

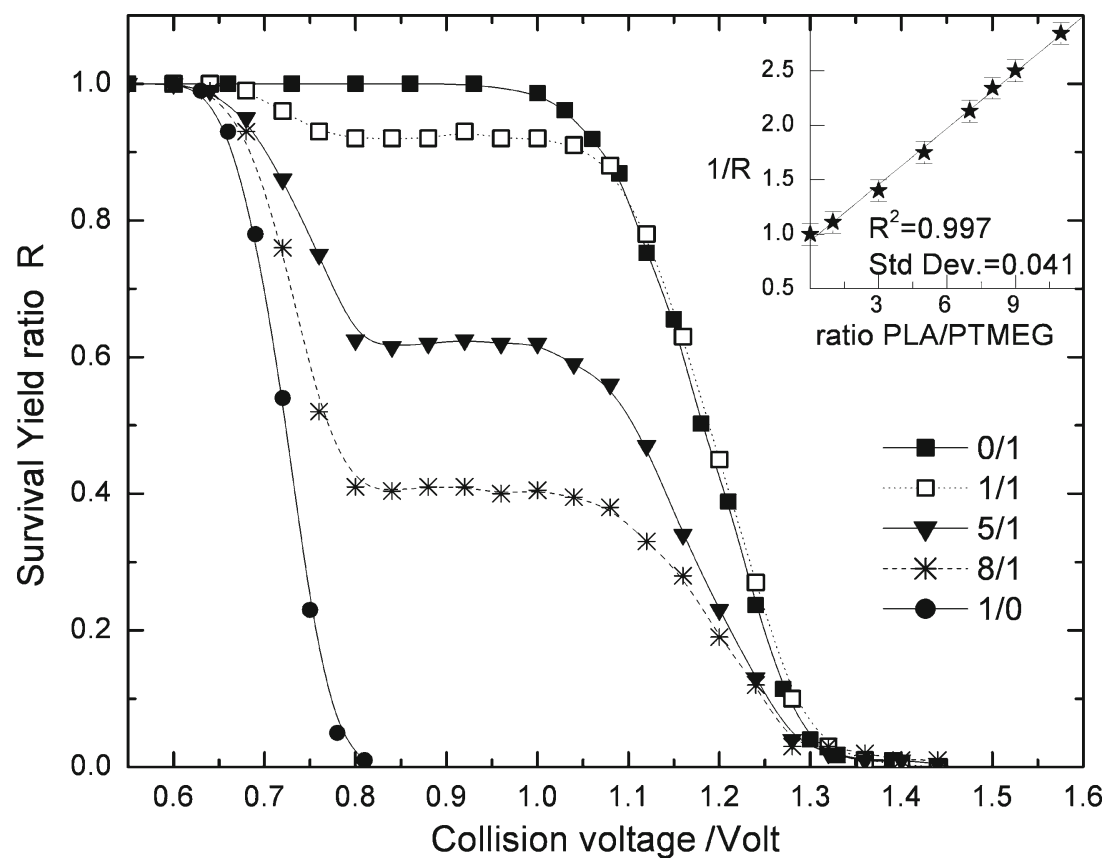

Figure 3. SY curves for PLA/PTMEG mixtures at relative concentrations $0: 1,1: 1,5: 1,8: 1$, and 1:0, in the same experimental conditions. The inset shows the correlation between the inverse of the SY ratio at the plateau (R) and the ratio PLA/PTMEG from sample preparation $0: 1,1: 1,5: 1,7: 1,8: 1,9: 1$, and $11: 1$ 
for quantitative purposes using, e.g., the standard addition method or a priori calibration. In order for these observations to be more practical, one may use the following strategy: at first, the compound of interest must be studied by means of drawing SY curve for the existence of a plateau to be evidenced. This demonstrates the isobaric contamination. Then, MS/MS spectra must be acquired at a single voltage corresponding to the intermediate regime (at the plateau) for different concentrations of the analyte or of the contaminant. The monitoring of the SY ratio at this unique excitation voltage at different concentrations, allows for drawing a titration curve as presented in the inset of Figure 3. This way, the drawing of multiple SY curves, which is rather time-consuming, is avoided and quantification can be quickly obtained following an experimental sequence similar to the standard addition method.

For quantitative purposes, it is important to have an evaluation of the performance of the proposed methodology. To this end, we have performed stability as well as a repeatability analysis of tandem MS spectra acquired for the mixture at an excitation voltage of $0.9 \mathrm{~V}$ (at the middle of the plateau). The same spectrum has been recorded separately 10 times, successively, in the same experimental conditions and following the same methodology (acquisition and data treatment methods) on three different days. From the combination of these stability and repeatability analyses, we obtained at most $2 \%$ deviation from the average value.

Using this quantitative estimate, one may try recovering "pure" MS/MS spectra for both homopolymers at any excitation voltages using information obtained at the intermediate excitation regime. We have observed that by applying simple spectral subtraction using Eqs. S-1 or S-2 according to the excitation regime (see Supplementary Material), corrected SY curves compare very well to SY curves for pure analytes. These results must be combined with the fact that the position of the plateau scales linearly with the relative quantities of the two components (see above). By doing so, SY curve of the mixture can be considered as a linear combination of the curves of the two analytes along the y-axis, while it is not sensitive to contamination along the x-axis. Simply, the nature and quantity of molecular ions do not affect the fragmentation energetic behavior of the other isobaric compounds and, at constant experimental conditions, the shape and position of the SY curve characterize the compound. However, details of MS/MS spectra, more precisely peaks relative intensities, are significantly different. The latter results demonstrate that consecutive fragmentations constitute an important part of the fragmentation processes in our experimental conditions. Details of this part of the study, containing graphs and formulas, are presented in the Supplementary Material.

\section{Size Effect on the Separation Power}

The technique presented requires that isobars fragment at different excitation voltages. In other terms, parameters associated with the fragmentation processes observed should have sufficiently different values to observe a plateau (intermediate regime). We have recently demonstrated [15] the collision energy necessary to achieve a certain degree of fragmentation increases linearly with the mass to a very good approximation $\left(\mathrm{R}^{2}>0.996\right)$. This analysis was performed on another polyether, namely poly(ethylene glycols), and tested on a wide variety of conditions (different instruments, data statistical treatment, experimental conditions ...). Moreover, when structurally different compounds are studied, the same linearity is observed but with different rate of increase [19]. Combining these two observations suggests the shift of the SY curves observed for isobars would increase with their mass providing the fragmentation parameters remain independent of the molecular ion mass. Consequently, the width of the intermediate regime observed in Figure 2 must increase with masses of the isobars due to molecular ions masses (as a consequence of the degrees of freedom effect and invariability of fragmentation parameters $[15,22])$. To assess this prediction, we have reproduced the SY curves as presented in Figure 2 for lithium cationized PLA and PTMEG with polymerization degree varying from 14 up to 23 (from approximately $\mathrm{m} / \mathrm{z} 1000$ up to $\mathrm{m} / \mathrm{z} 1700$ ) under the same experimental conditions (see Figure 4). The widths of the plateaus, which may be called the isobaric resolving power, have been estimated using SY curves for pure homopolymers, considering voltages necessary to achieve more than $99 \%$ fragmentation of $\left[\mathrm{PLA}_{23}+\mathrm{Li}\right]^{+}$and less than $1 \%$ fragmentation of $\left[\mathrm{PTMEG}_{23}+\mathrm{Li}\right]^{+}$. Error bars were overestimated, considering $0.02 \mathrm{~V}$ uncertainties, when determining the latter voltages. The linear fit is in agreement with our expectation and demonstrates that the resolving power of the technique can increase significantly with molecular ion mass (the width is doubled between $\mathrm{m} / \mathrm{z} 1000$ and $\mathrm{m} / \mathrm{z}$ 1700). Considering the fragmentation parameters remain constant with respect to the polymerization degree, this technique allows for an efficient

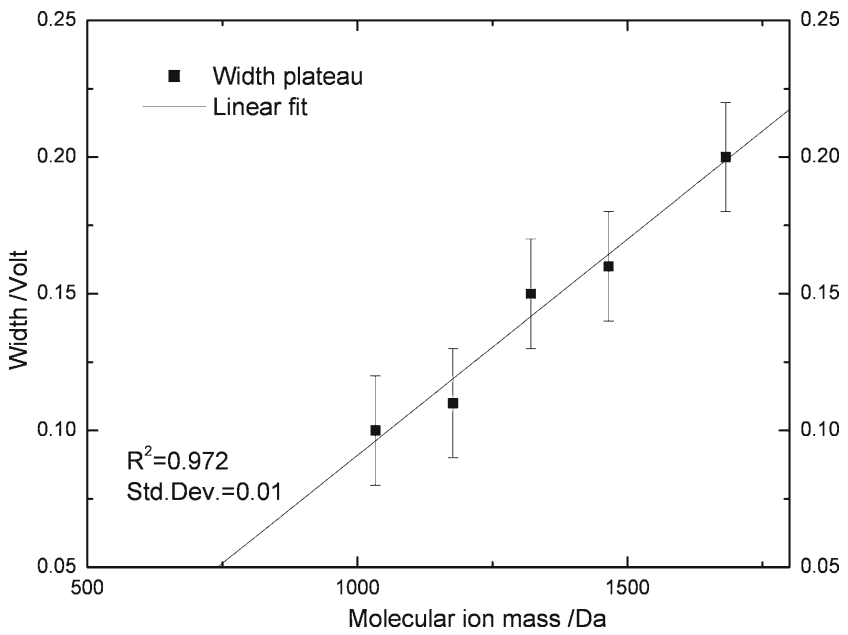

Figure 4. Width of the plateau (intermediate regime) as a function of molecular ions mass for a mixture of $\left[\mathrm{PLA}_{23}+\mathrm{Li}\right]^{+} /$ $\left[\mathrm{PTMEG}_{23}+\mathrm{Li}\right]^{+}$. Details on calculations and estimation of error bars are presented in the text 
separation of isobars and may enable refined structural characterization according to the fragmentation parameters at large $\mathrm{m} / \mathrm{z}$. If the linearity observed can be explained as a consequence of the degrees of freedom effect, the negative intercept suggests that PLA/PTMEG mixtures with masses below $m / z 500$ cannot be resolved. The negative values are associated with the fact that $\left[\mathrm{PTMEG}_{\mathrm{n}}+\mathrm{Li}\right]^{+}$would simply start fragmenting before $\left[\mathrm{PLA}_{\mathrm{n}}+\mathrm{Li}\right]^{+}$has fully decomposed. Obviously, experimental conditions may modify these features; the mass-limit separation may be shifted to higher or lower masses, and the resolving power increased at the same molecular ion mass.

\section{Limits of the Method}

For a mixture of compounds with very similar fragmentation parameters and low $\mathrm{m} / \mathrm{z}$, one would expect no intermediate regime and a SY curve with a modified shape but without any plateau. Trying to satisfy those two conditions, we have studied two protonated peptides with inverted sequences, $\mathrm{H}-$ (SDGRGAF)- $\mathrm{NH}_{2}$ (reverse 1) and $\mathrm{H}$-(FAGRGDS)- $\mathrm{NH}_{2}$ (reverse 2), separately and in a 1:1 mixture. Due to little structural differences, fragmentation parameters have a good chance to be also very close. Moreover, those peptides are exact isobars with $m / z 708.3$, which is very close to the resolving limit identified for PLA/PTMEG mixtures (for which, in addition, fragmentation parameters may be significantly more different). Results are presented in Figure 5. As the first component has just started to fragment, the second component is seemingly fragmenting as well. Thus, concerning the equimolar mixture, the SY ratio as a function of excitation voltage consists of a curve with a continuous and smooth decrease but without any plateau, a shape that is expected for pure analytes. For an unknown mixture of isobars with a similar behavior, visual inspection of tandem mass spectra does not help identifying the contamination; neither does the analysis of spectra at low and at high excitation voltages. MS/MS spectra are contaminated at all excitation voltages and, consequently, do not provide structural information on one compound only but rather give mixtures of fragments from both isobars. In the absence of such a plateau, identifying contamination, even qualitatively only, is not possible. This also illustrates the limit of the conventional comparison between tandem mass spectra obtained at high and at low energies for structural investigation of isobars. In this respect it is, however, worth noting that the curve for the mixture is located in a position that is intermediate to the two curves obtained for pure compounds. Modification of the SY curve shape or location seems to be a possible qualitative indicator of isobaric contamination, but this requires a priori knowledge of the curve for at least one of the two pure components. Indeed, at constant experimental conditions, the SY curve for a given analyte has a shape and position that depend on the molecular mass as well as on the parameters associated to the fragmentation processes observed. These processes in turn depend on the details of the structure of the analyte, making the SY curve a kind of fingerprint of the compound. Thus, any deviation from the determined curve, in the same experimental conditions, demonstrates the presence of an isobaric contaminant.

With the SY curves of the two components at hands, we have tried to estimate the degree of contamination using a simple weighted combination. This way we could easily achieve at most $5 \%$ deviation from measured values. Such deviations, however, do not compare favorably to the shift

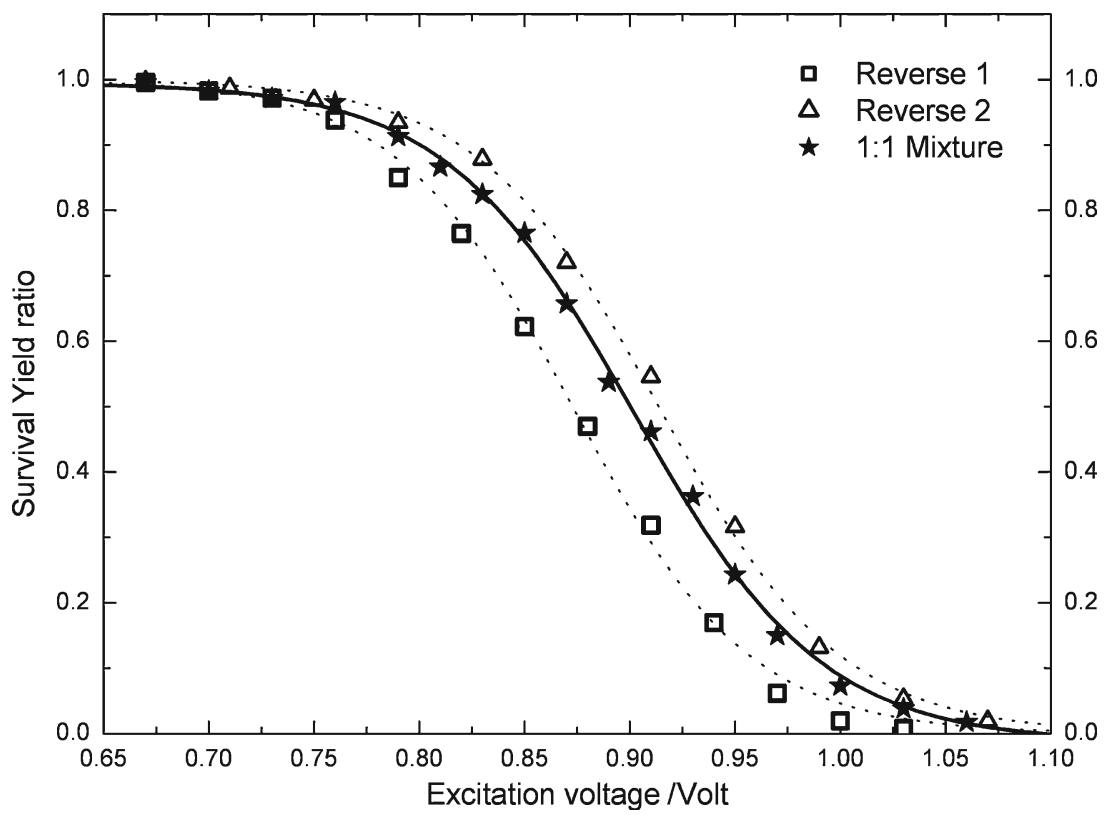

Figure 5. Survival Yield curves for two protonated peptides with inverted sequences at $m / z$ 708.3: H-(SDGRGAF)-NH 2 (reverse 1) and $\mathrm{H}$-(FAGRGDS)- $\mathrm{NH}_{2}$ (reverse 2). SY curve for a 1:1 mixture is also presented 
of SY curves as they represent $10 \%$ to $40 \%$ of the corresponding distances between SY curves for pure protonated peptides. Although not very accurate at this stage, the method seems nonetheless promising for quantitative evaluation of isobars even in the absence of a plateau. The combination of low $\mathrm{m} / \mathrm{z}$ and small differences in the fragmentation parameters leads to a negative value for the resolving power and thus, necessitates suspicion of isobaric contamination and a priori knowledge of the structure of one of the two components to unequivocally decide on the contamination.

\section{Conclusions}

Results presented in this work show the Survival Yield curve of an isobaric mixture consists in a linear combination of survival yield curves for the two compounds separately. Moreover, the survival yield curve for a mixture was shown to exhibit a plateau. Combining the latter two observations led to the following conclusions: (1) the existence of a plateau demonstrates that the sample is made up of a mixture of compounds and allows for studying the structure of the two components separately and unambiguously, and (2) the vertical position of the plateau can be used for quantifying the two components of the mixture, and there is no need for a priori knowledge of the respective fragmentation patterns. These results are, however, limited to compounds with sufficiently different fragmentation parameters, where "sufficiently" is not well-defined and is a priori not restrictive, as it may partly be compensated by the size effect. This is welldemonstrated by the increase in the width of the plateau as $\mathrm{m} / \mathrm{z}$ becomes larger.

Theoretically, the existence of such a plateau was never mentioned in the literature, and is a new feature of the survival yield technique that may lead to a better understanding of its physical and chemical information content (e.g., from the collisional or ionization processes).

From practical point of view, the presented methodology for quantitation may be particularly interesting as polyethers are present in perfumes, plastic containers, and also in various laboratory consumables. They are thus potential contaminants even during an analytical procedure. Using the proposed methodology, they may be easily traced for isobaric contamination even for large mass compounds. These synthetic oligomers are, indeed, known to fragment at much higher energies than biological compounds $[15,19$, 22] that allow for using the methodology presented in this work. Similarly, polyethers are extensively used in packaging and must thus be tracked in, e.g., foodstuff, for possible contamination. Alternatively, after an intermediate excitation regime, namely a plateau, has been identified, the methodology can be used for structural study and quantification of a compound of interest without the need for cleaning the sample from isobaric polyethers: this way, the purity of an analyte can be determined using MS/MS and the
Survival Yield technique only, without necessarily using complex and tedious sample preparation or cleaning.

\section{Acknowledgments}

Département de Chimie Moléculaire (DCM), Université Joseph Fourier is gratefully acknowledged for financial support. The authors thank Julien De Winter (UMONS, Mass Spectrometry Research Group, Mons, Belgium) for kindly providing them with samples of poly(lactic acid), and Rodolphe Guéret for technical assistance. A.M. also thanks Professor K. Vekey and Dr. L. Drahos for support and useful suggestions and Professor J. C. Tabet for careful reading of the manuscript.

\section{References}

1. Zhu, P., Bowden, P., Zhang, D., Marshall, J.G.: Mass spectrometry of peptides and proteins from human blood. Mass Spectrom. Rev. EarlyView (2010). doi:10.1002/mas.20291

2. Hughey, C.A., Rodgers, R.P., Marshall, A.G.: Resolution of 11000 compositionally distinct components in a single electrospray ionization Fourier transform ion cyclotron resonance mass spectrum of crude oil. Anal. Chem. 74, 4145-4149 (2002)

3. Purcell, J.M., Hendrickson, C.L., Rodgers, R.P., Marshall, A.G.: Atmospheric pressure photionization Fourier transform ion cyclotron resonance mass spectrometry for complex mixture analysis. Anal. Chem. 78, 5906-5912 (2006)

4. Gill, L.A., Wells, J.M., Patterson, G.E., Amy, J.W., Cooks, R.G.: Resolution of isobaric and isomeric ions using chemical shifts in an ion trap mass spectrometer. Anal. Chem. 70, 4448-4452 (1998)

5. Schnier, P.D., Williams, E.R.: Analysis of isomeric mixtures using blackbody infrared Radiative dissociation: Determining isomeric purity and obtaining individual tandem mass spectra simultaneously. Anal. Chem. 70, 3033-3041 (1998)

6. Kushnir, M.M., Rockwood, A.L., Nelson, G.J.: Simultaneous quantitative analysis of isobars by tandem mass spectrometry from unresolved chromatographic peaks. J. Mass Spectrom. 39, 532-540 (2004)

7. Barnes, C.A.S., Hilderbrand, A.E., Valentine, S.J., Clemmer, D.E.: Resolving isomeric peptide mixtures: a combined HPLC/ion mobilityTOF-MS analysis of a 4000-component combinatorial library. Anal. Chem. 74, 26-36 (2002)

8. Beres, T., Zatloukal, M., Voller, J., Niemann, P., Gahsche, M.C., Tarkowski, P., Novak, O., Hanus, J., Strnad, M., Dolezal, K.: Tandem mass spectrometry identification and LC-MS quantification of intact cytokinin nucleotides in K-562 human leukemia cells. Anal. Bioanal. Chem. 398, 2071-2080 (2010)

9. Tao, W.A., Wu, L., Cooks, R.G.: Differentiation and quantitation of isomeric dipeptides by low-energy dissociation of copper(II)-bound complexes. J. Am. Soc. Mass Spectrom. 12, 490-496 (2001)

10. Majumdar, T.P., Clairet, F., Tabet, J.C., Cooks, R.G.: Epimer distinctions and structural effects on gas phase acidities of alcohols measured using the kinetic method. J. Am. Chem. Soc. 114, 2897-2903 (1992)

11. Wu, L., Lemr, K., Aggerholm, T., Cooks, R.G.: Recognition and quantification of binary and ternary mixtures of isomeric peptides by kinetic method: metal ion and ligand effects on the dissociation of metal-bound complexes. J. Am. Soc. Mass Spectrom. 14, 152 (2003)

12. Desaire, H., Leary, J.A.: Multicomponent quantification of diastereomeric hexosamine monosaccharides using ion trap tandem mass spectrometry. Anal. Chem. 71, 4142 (1999)

13. De Winter, J., Lemaur, V., Marsal, P., Coulembier, O., Cornil, J., Dubois, P., Gerbaux, P.: Mechanistic study of the collision-induced dissociation of sodium-cationized polylactide oligomers: A joint experimental and theoretical investigation. J. Am. Soc. Mass Spectrom. 21, 1159-1168 (2010)

14. Kuki, A., Nagy, L., Memboeuf, A., Drahos, L., Vekey, K., Zsuga, M., Keki, S.: Energy-dependent collision-induced dissociation of lithiated polytetrahydrofuran: Effect of the size on the fragmentation properties. J. Am. Soc. Mass Spectrom. 21, 1753-1761 (2010) 
15. Memboeuf, A., Nasioudis, A., Indelicato, S., Pollreisz, F., Kuki, A., Keki, S., van den Brink, O.F., Vekey, K., Drahos, L.: Size effect on fragmentation in tandem mass spectrometry. Anal. Chem. 82, 2294$2302(2010)$

16. Derwa, F., Pauw, E.D., Natalis, P.: New basis for a method for the estimation for secondary ion internal energy distribution in 'soft' ionization techniques. Org. Mass Spectrom. 26, 117-118 (1991)

17. Guo, X.H., Duursma, M.C., Kistemaker, P.G., Nibbering, N.M.M., Vekey, K., Drahos, L., Heeren, R.M.A.: Manipulating internal energy of protonated biomolecules in electrospray ionization Fourier transform ion cyclotron resonance mass spectrometry. J. Mass Spectrom. 38, 597-606 (2003)

18. Collette, C., Drahos, L., Pauw, E.D., Vekey, K.: Comparison of the internal energy distribution of ions produced by different electrospray sources. Rapid Commun. Mass Spectrom. 12, 1673-1678 (1998)
19. Nasidoudis, A., Memboeuf, A., Heeren, R.M.A., Smith, D.F., Vekey, K., Drahos, L., van den Brink, O.F.: Discrimination of polymers by using their characteristic collision energy in tandem mass spectrometry. Anal. Chem. 82, 9350-9356 (2010)

20. Haller, I., Mirza, U.A., Chait, B.T.: Collision induced decomposition of peptides. Choice of collision parameters. J. Am. Soc. Mass Spectrom. 7, 677-681 (1996)

21. Kertesz, T.M., Hall, L.H., Hill, D.W., Grant, D.F.: $\mathrm{CE}_{50}$ : Quantifying collision induced dissociation energy for small molecule characterization and identification. J. Am. Soc. Mass Spectrom. 20, 1759-1767 (2009)

22. Memboeuf, A., Drahos, L., Vekey, K., Lendvay, G.: Energetics of fragmentation for cationized poly(ethylene glycol) oligomers. Rapid Commun. Mass Spectrom. 24, 2471-2473 (2010) 